\title{
Relation between development of cardiovascular disease and the C242T CYBA polymorphism of the NADPH oxidase in ESRD patients
}

\author{
Feng-Ying Tang ${ }^{\mathrm{a}, 1, *}$, Ying Zhu ${ }^{\mathrm{a}}$, Gui Hua Wang ${ }^{\mathrm{a}}$ and Xiong-Wei Xie ${ }^{\mathrm{b}}$ \\ ${ }^{a}$ Department of Nephrology, Affiliated the No.1 People's Hospital of Huaian, Nanjing Medical university, Huaian, \\ Jiangsu, P.R. China \\ ${ }^{\mathrm{b}}$ Department of Cardiology, Affiliated the No.1 People's Hospital of Huaian, Nanjing Medical university, Huaian, \\ Jiangsu, P.R. China
}

\begin{abstract}
Background: The development of cardiovascular disease in ESRD patients is considered to be associated with oxidative stress. $\mathrm{NAD}(\mathrm{P}) \mathrm{H}$ oxidase has attracted attention as mechanisms of generating oxidative stress. We investigated the relation between the genotype of the C242T CYBA polymorphism of the NADPH oxidase and the development of cardiovascular disease in ESRD patients.

Methods: A total of 289 ESRD patients were recruited and allocated to one of the two groups: patients without cardiovascular disease (group $\mathrm{N} ; n=192$ ) and patients developing cardiovascular disease (group $\mathrm{D} ; n=97$ ). The C242T CYBA polymorphism was determined by RFLP-PCR methods.

Results: The frequency of the C242T CT+TT genotype was significantly lower in group D than in group N (9.1 vs. 20.2\%). In multiple Logistic regression analysis, systolic blood pressure, smoking history and this gene polymorphism were shown to be independent variables for the development of cardiovascular disease in ESRD patients.

Conclusions: These results suggest that assessment of the C242T CYBA polymorphism of the NADPH oxidase may be useful in identifying the risk for developing cardiovascular disease in ESRD patients.
\end{abstract}

Keywords: NAD(P)H Oxidase, gene polymorphism, cardiovascular disease

\section{Introduction}

Despite significant progress in renal replacement therapy, the mortality from cardiovascular disease (CVD) in patients with end-stage renal failure (ESRD) is many times higher than in the general population. Although the traditional risk factors are frequently present

\footnotetext{
* Corresponding author: Feng-Ying Tang, Department of Nephrology, Affiliated the No.1 People's Hospital of Huaian, Nanjing Medical university, Huaian, Jiangsu, P.R. China. The Beijing Xi Road No.6, Huaian, 223300, Jiangsu, P.R. China. E-mail: tangfengying1976@yahoo.com.cn.

${ }^{1}$ Supported by the Postdoctoral Scientific and technological Project of Hunan Province.
}

in ESRD patients, these factors do not fully explain the extraordinary increase in morbidity and mortality in CVD among patients with ESRD. Recent studies have highlighted the accumulation of reactive oxygen species (ROS) as one of the mechanism involved in the development of CVD of ESRD patients [1], via the following pathological pathways: ROS may injure the endothelial cell membrane, inactivate NO, and cause oxidation of an essential cofactor of nitric oxide synthase (NOS) $[2,3]$. It has been proposed that genes associated with oxidative stress contribute to the risk of CVD in ESRD patients. Recently, NADPH oxidase has received attention as mechanisms of generating oxidative stress. Catalyzing the 1-electron reduction of oxygen using $\mathrm{NAD}(\mathrm{P}) \mathrm{H}$ as the electron donor, NADH/NADPH 
oxidase is a membrane-associated enzyme for superoxide production in vascular smooth muscle cells and endothelial cells [4]. Studies indicate that NAD(P)H oxidase is the most important source of ROS in intact arteries [5-8]. In addition, it has reported that the C242T CYBA polymorphism of the NADPH oxidase is associated with vascular superoxide production in human blood vessels from CVD patients [9]. Therefore, it is possible that this polymorphism may contribute to the imbalance of ROS found in CVD in ESRD patients. In this study, we decide to investigate the association of genotype of the susceptibility to developing CVD in Chinese ESRD patients.

\section{Materials and methods}

\subsection{Subjects}

The subjects were 289 unrelated Chinese patients diagnosed with ESRD who were recruited in the outpatient clinic and inpatient department of the second $\mathrm{Xi}$ angya Hospital of Central South University since 2005. From these subjects, we allocated them to one of the two groups: group N, comprised of 192 patients without CVD, and group D, comprised of 97 patients with CVD. Of the 97 ESRD patients with CVD, 26 patients had experienced a myocardial infarction, and 71 patients had angina pectoris diagnosed by an exercise test or by scintigraphy. Group N was defined using the following criteria: 1) all participants had creatinine clearance of $<10 \mathrm{ml} / \mathrm{min}$ for 5 years and 2) subjects with diagnosis of CVD according to the following criteria were excluded. Group D was defined according to the following criteria: 1) all participants had creatinine clearance of $<10 \mathrm{ml} / \mathrm{min}$ for 5 years and 2) a previous myocardial infarction was defined as a heart attack diagnosed by a physician based on chest pain, electrocardiographic changes, and enzyme determinations or the results of coronary angiography. In patients with chest pain and ischemic electrocardiographic abnormalities, an exercise test or dipyridamole thallium scintigraphy was performed. Patients with a history of myocardial infarction or a positive result in one of these tests were diagnosed with coronary artery disease [10].

Clinical data, including onset age, BMI at the diagnosis of ESRD, history of hypertension, blood pressure, history of smoking and lipid profiles were obtained from the medical records of the patients. BMI was calculated as body weight in kilograms divided by the square of body height in meters. Body weight and body height were measured with light clothes and bare feet. The systolic and diastolic blood pressures used in the analysis were the average of three sitting blood pressure readings. Smoking history was defined on the basis of current cigarette smoking or previous habitual smoking. After at least an 8-h fast, blood samples were collected from the subjects, and serum total cholesterol, LDL cholesterol, HDL cholesterol, creatinine, and triglyceride were determined by at the Nephrology Laboratories of the second Xiangya Hospital of Central South University following standard laboratory protocols. LDL cholesterol was calculated using the Friedewald formula. Serum creatinine was performed with a Beckman CXR rate method using the Jaffe reaction. Creatinine clearance calculated according to the Cockcroft-Gault formula.

Each patient was diagnosed by at least two physicians on the basis of unstructured interviews and information from medical records, and the Kappa coefficients of agreement between raters were 0.91. All participants belong to the Han ethnic group in Hunan Province. The local ethical committee approved the experimental design, and all participants gave their informed consent.

\subsection{Genotyping}

Genomic DNA was extracted from peripheral blood cells using a DNA extraction kit (PUREGENE ${ }^{\mathrm{TM}}$ Blood Kit; Gentra) and the DNA concentration was then determined by spectrophotometry. The C242T polymorphism was determined by PCR and $R s a \mathrm{I}$ digestion.

The DNA fragment containing the polymorphic site was amplified from genomic DNA by PCR with sense oligonucleotide primer 5' - CTC TGT GTT GTC TTC AGT AAA GG-3' and antisense oligonucleotide primer 5'-ACT CAC AGG AGA TGC AGG ACG-3' [11]. The $50 \mu \mathrm{l}$ reaction mixture contained $0.1 \mu \mathrm{g}$ of genomic DNA; $5 \mu$ l of $10 \times$ Taq buffer with $\mathrm{Kcl}$ ( MBI, fermentas ); $4 \mu \mathrm{l}$ of $25 \mathrm{mM} \mathrm{MgCl}_{2}$ solution; $2 \mu \mathrm{l}$ each of the $10 \mu \mathrm{M}$ sense and antisense primers; $1 \mu \mathrm{l}$ of $10 \mathrm{mM}$ deoxy-nucleotide triphosphates, specifically deoxy-adenosine triphosphate, guanosine triphosphate, cytosine triphosphate, and thymidine triphosphate; and 1.25U of Taq DNA polymerase. The PCR consisted of the initial denaturation step for $5 \mathrm{~min}$ at $94^{\circ} \mathrm{C}, 32 \mathrm{cy}-$ cles of denaturation for $30 \mathrm{~s}$ at $92^{\circ} \mathrm{C}$, primer annealing for $45 \mathrm{~s}$ at $62^{\circ} \mathrm{C}$, and primer extension for $90 \mathrm{~s}$ at $72^{\circ} \mathrm{C}$, followed by a final extension step for $10 \mathrm{~min}$ at $72^{\circ} \mathrm{C}$. After digestion with $R s a \mathrm{I}$ (MBI, Fermentas), the 
Table 1

Clinical characteristics of ESRD patients with CVD and without

\begin{tabular}{lcc}
\hline Variables & Group $\mathrm{D}(n=97)$ & Group $\mathrm{N}(n=192)$ \\
\hline Age $(\mathrm{yr})$ & $54.4 \pm 11.5$ & $53.3 \pm 12.6$ \\
sex(M/F) & $50 / 47$ & $99 / 93$ \\
BMI & $23.2 \pm 2.1$ & $24.1 \pm 3.0$ \\
Hypertension(yes/no) & $57 / 40$ & $110 / 82$ \\
SBp $(\mathrm{mmHg})$ & $160.1 \pm 26.42^{\mathrm{a}}$ & $115.2 \pm 21.21$ \\
$\mathrm{DBp}(\mathrm{mmHg})$ & $81.23 \pm 17.1$ & $80.98 \pm 15.9$ \\
Smoking history(yes/no) & $60 / 37^{\mathrm{a}}$ & $90 / 102$ \\
Total cholesterol(mmol/L) & $5.20 \pm 0.66$ & $5.12 \pm 1.01$ \\
Triglycerides(mmol/L) & $1.58 \pm 0.99$ & $1.57 \pm 1.09$ \\
HDL cholesterol(mmol/L) & $1.01 \pm 0.32$ & $1.11 \pm 0.23$ \\
LDL cholesterol(mmol/L) & $3.12 \pm 1.31$ & $3.11 \pm 1.03$ \\
\hline${ }^{\mathrm{a}} p<0.05$ versus controls. & &
\end{tabular}

Table 2

Frequencies of genotypes of p22phox C242T polymorphism

\begin{tabular}{lcccc}
\hline Genotype & Group $\mathrm{D}(n=97)$ & Group N $(n=192)$ & $\chi^{2}$ & $p$ \\
\hline CC & $87(89.7)$ & $152(79.2)$ & & \\
CT+TT & $10(10.3)$ & $40(20.8)$ & 4.988 & 0.026 \\
\hline
\end{tabular}

Numbers in parentheses indicate percentages.

samples were separated on $1.5 \%$ agarose gel and visualized with ethidium bromide. Digestion of the PCR products yielded bands of $348 \mathrm{bp}$ in CC homozygotes, 188 and $160 \mathrm{bp}$ in TT homozygotes, and all three bands in heterozygotes. Each genotype was read by two individuals independently; if in conflict, genotyping was repeated.

\subsection{Statistical analysis}

Hardy-Weinberg Simulator software (http://ihg2. helmholtz-muenchen.de/cgi-bin/hw/hwal.pl) was used to test the genotype distribution for Hardy-Weinberg equilibrium. Data were expressed as means $\pm \mathrm{SD}$. Student's t test and chi-square test were used to compare the differences of the continuous and categorical variables between groups. $\chi^{2}$ analysis was used to compare the genotype distributions of genotypes and alleles between groups. Logistic regression analyses were used to adjust for possible confounders including age, sex, BMI, smoking habit, history of hypertension, SBP and DBP and evaluate the OR of the genotypes and alleles for ESRD patients with CVD and their $95 \%$ confidence interval (CI) with two-tailed $p$ values. All statistical analysis was performed using SPSS 13.0.

\section{Results}

Table 1 shows the clinical characteristic of the enrolled patients. Clinical data, including age, sex, BMI, history of hypertension, blood pressure, history of smoking, and lipid profiles were obtained from the medical records. There were no significant differences in sex, age, BMI, history of hypertension, diastolic blood pressures, serum total cholesterol, HDL, LDL cholesterol, and triglyceride between group $\mathrm{N}$ and $\mathrm{D}$, and the systolic blood pressure and smoking history of group D were significantly higher than that of group N.

The genotype distribution of this gene in each group is shown in Table 2. The genotype frequencies in both groups were in the Hardy-Weinberg equilibrium. Because the TT genotype frequency was low, we divided the enrolled subjects into two groups: CC and CT $+\mathrm{TT}$. The frequency of the CC, CT+TT genotypes of the p22phox gene were 79.2 and $20.8 \%$ in group $\mathrm{N}$ compared with 89.7 and $10.3 \%$ in group D, respectively. The frequency of the CT+TT genotypes was significantly higher in group $\mathrm{N}$ than in group $\mathrm{D}\left(\chi^{2}=4.988\right.$, $p=0.026$ ).

The risk factors for the development of CVD in the ESRD patients are shown in Table 3. Logistic regression analysis identified systolic blood pressure and smoking history as independent risk factors for CVD of ESRD within the following variables: age, sex, body mass index (BMI), diastolic blood pressure (DBP), history of hypertension, smoking history, serum total cholesterol, HDL, LDL cholesterol, and triglyceride levels. When the CT+TT genotypes were introduced into this model, the CT+TT genotypes were an independent protective factor for CVD in the ESRD patients: adjusted odds ratio 0.423 (95\% CI 0.239-0.711, 
Table 3

Risk of CVD in ESRD patients

\begin{tabular}{lccc}
\hline Variables & Adjusted odds ratio & $95 \%$ CI & $P$ value \\
\hline Age(yr) & 0.912 & $0.318-1.476$ & 0.721 \\
Gender(M/F) & 1.001 & $0.880-1.234$ & 0.113 \\
BMI & 1.002 & $0.894-1.345$ & 0.132 \\
Hypertension(\%) & 1.002 & $0.768-1.612$ & 0.881 \\
SBp $(\mathrm{mmHg})$ & 1.997 & $1.038-3.711$ & 0.021 \\
DBp(mmHg) & 1.107 & $0.499-2.411$ & 0.317 \\
Smoking history(\%) & 2.081 & $1.413-4.478$ & 0.004 \\
Total cholesterol(mmol/L) & 1.043 & $0.472-1.898$ & 0.612 \\
Triglycerides(mmol/L) & 1.004 & $0.521-1.988$ & 0.781 \\
HDL cholesterol(mmol/L) & 0.871 & $0.234-1.891$ & 0.478 \\
LDL cholesterol(mmol/L) & 1.011 & $0.201-1.991$ & 0.572 \\
CC & 2.391 & $1.101-4.289$ & 0.004 \\
CT+TT & 0.423 & $0.239-0.711$ & 0.011 \\
\hline
\end{tabular}

Logistic regression analysis taking into account of age, gender, BMI, Hypertension, SBp, DBp, Total cholesterol, Triglycerides, HDL cholesterol and LDL cholesterol. The concordance between the observed and predicted risk of CVD in ESRD patients was $57.9 \%$.

$P=0.011)$. These analyses revealed that this polymorphism is a significant factor in the development of CVD.

\section{Discussion}

In this study, we investigated the relationship between CVD in ESRD patients and the C242T polymorphism and found that the $242 \mathrm{~T}$ allele is associated with significantly lower risk of CVD in ESRD patients. These findings provide direct data relating CVD in ESRD patients to the C242T polymorphism.

NAD(P)H oxidase is a critical enzyme for superoxide production in phagocytes, vascular smooth muscle cells, and mesangial cells [4]. P22phox is an essential component of NADPH oxidase. The C242T polymorphism results in an amino acid polymorphism (His/Tyr) at residue 72 of p22phox involving a potential hemebinding site [12]. Because the histidine residue is considered to be a candidate for the ligand of the heme prosthetic group of cytochrome $b$, it has been revealed that this polymorphism is associated with the function of p22phox and significantly lower basal and NADPHstimulated vascular superoxidase production in human blood vessels from patients with atherosclerosis [13, 14]. Individuals with the $242 \mathrm{~T}$ genotype might have lower oxidative stress as a result of lower $\mathrm{O}_{2}^{-}$production compared with individuals bearing the $242 \mathrm{C}$ genotype.

Previous studies have examined this polymorphism in association with CVD. Inoue et al. found that the $242 \mathrm{~T}$ allele was associated with a reduced risk of coronary artery disease [9], whereas other studies showed conflicting findings in patients with CVD and cerebrovascular disease [15-20]. However, the genotyping of patients in the prospective Lipoprotein and Coronary Artery Study (LCAS) suggested a significant association between the $242 \mathrm{~T}$ allele and the progression of coronary atherosclerosis [17]. At the same time, studies on this polymorphism in association with CKD have produced conflicting results. Matsunaga-Irie et al. found a significant association with diabetic nephropathy in type 2 diabetes [21]. Hodgkinson et al. showed an association of the $\mathrm{C} 242 \mathrm{~T}$ polymorphism with diabetic nephropathy in type 1 diabetes [22]. However, Wolf et al. could not demonstrate any significant association of the C242T polymorphism with IgA nephropathy and lupus nephritis [23]. Doi et al. found that the T allele of the C242T polymorphism showed a protective effect against ESRD only in the nondiabetic (non-DM) group [24]. We performed a case-control association study consisting of a total of 97 cases and 192 control samples to elucidate the polymorphism in the CYBA gene encoding NAD $(\mathrm{P}) \mathrm{H}$ p22 phox and identified a strong association of the CT+TT genotype of the C242T polymorphism with CVD in ESRD even after adjusting for confounding factors.

The conflicting results may reflect the ethnic backgrounds of the study populations. Inadequacy in the sample size, genetic heterogeneity, environmental background, and different definition of renal disease phenotype are additional limited factors. We have attempted to minimize these pitfalls by selecting wellmatched samples from one single center. Moreover, our population can be considered fairly homogeneous, with all subjects originating from the same geographically well-defined catchment area (city of Hunan Province 
of China). ESRD with CVD phenotype was also defined strictly. In our study, adhering to a case-control association strategy, we found that patients with the $\mathrm{CT}+\mathrm{TT}$ genotype have a lower risk of developing CVD in ESRD. After adjusting for the confounding factors in Logistic regression model, the CT+TT genotypes were an independent protective factor for CVD in the ESRD patients.

In conclusion, we propose that the C242T CYBA polymorphism of the NADPH oxidase is associated with the development of CVD in ESRD. To further elucidate the potential association between the C242T CYBA polymorphism of the NADPH oxidase and CVD in ESRD patients, investigations with larger sample size are needed to confirm these associations.

\section{References}

[1] Z.D. Vaziri, Z. Ni, F. Oveisi, K. Liang and R. Pandian, Enhanced nitric oxide inactivation and protein nitration by reactive oxygen species in renal insufficiency, Hypertension 39 (2002), 135-141.

[2] M. Annuk, M. Zilmer and B. Fellstrom, Endotheliumdependent vasodilation and oxidative stress in chronic renal failure: impact on cardiovascular disease, Kidney Int Suppl $\mathbf{8 4}$ (2003), S50-S53.

[3] P.S. Modlinger, C.S. Wilcox and S. Aslam, Nitric oxide, oxidative stress, and progression of chronic renal failure, Semin Nephrol 24(4) (2004), 354-365.

[4] M. Ushio-Fukai, A.M. Zafari, T. Fukui, N. Ishizaka and K.K. Griendling, P22phox is a critical component of the superoxidegenerating NADH/NADPH oxidase system and regulates angiotensin II-induced hypertrophy in vascular smooth muscle cells, J Biol Chem 271 (1996), 23317-23321.

[5] D. Sorescu, D. Weiss, B. Lassegue B et al., Superoxide production and expression of nox family proteins in human atherosclerosis, Circulation 105 (2002), 1429-1435.

[6] K.M. Mohazzab, P.M. Kaminski and M.S. Wolin, NADH oxidoreductase is a major source of superoxide anion in bovine coronary artery endothelium, Am J Physiol 266 (1994), H2568-H2572.

[7] K.K. Griendling, C.A. Minieri, J.D. Ollerenshaw and R.W. Alexander, Angiotensin II stimulates NADH and NADPH oxidase activity in cultured vascular smooth muscle cells, Circ Res 74 (1994), 1141-1148.

[8] S. Rajagopalan, S. Kurz, T. Munzel et al., Angiotensin IImediated hypertension in the rat increases vascular superoxide production via membrane NADH/NADPH oxidase activation: contribution to alterations of vasomotor tone, J Clin Invest 97 (1996), 1916-1923.

[9] N. Inoue, S. Kawashima, K. Kanazawa et al., Polymorphism of the NADH/NADPH oxidase p22phox gene in patients with coronary artery disease, Circulation 97 (1998), 135-137.

[10] H. Kimura, F. Gejyo, T. Yamaguchi et al., A cholesteryl ester transfer protein gene mutation and vascular disease in dialysis patients, J Am Soc Nephrol 10 (1999), 294-299.

[11] Y. Allanore, D. Borderie, H. Lemarechal et al., Lack of association of eNOS (G894T) and p22 phox NAPDH oxidase subunit (C242T) polymorphisms with systemic sclerosis in a cohort of French Caucasian patients, Clinica Chimica Acta (2004), 51-55.

[12] M.C. Dinauer, E.A. Pierce, G.A. Bruns, J.T. Curnutte and S.H. Orkin, Human neutrophil cytochrome b light chain(p22phox): gene structure, chromosomal location, and mutations in cytochrome-negative autosomal recessive chronic granulomatous disease, J Clin Invest 86 (1990), 1729-1737.

[13] M.S. Wolin, How could a genetic variant of the p22phox component of NADPH oxidases contribute to the progression of coronary atherosclerosis? Circ Res 86 (2000), 365-366.

[14] T.J. Guzik, N.E. West, E. Black et al., Functional effect of the $\mathrm{C} 242 \mathrm{~T}$ polymorphism in the NADPH oxidase p22phox gene on vascular superoxide production in atherosclerosis, Circulation 102 (2000), 1744-1747.

[15] A. Gardemann, P. Mages, N. Katz, Tillmanns and W. Haberbosh, The p22phox A640G gene polymorphism but not the $\mathrm{C} 242 \mathrm{~T}$ gene variation is associated with coronary heart disease in younger individuals, Atherosclerosis 145 (1999), 315-323.

[16] C. Cahilly, C.M. Ballantyne, D.S. Lim, A. Gotto and A.J. Marian, A variant of p22(phox), involved in generation of reactive oxygen species in the vessel wall, is associated with progression of coronary atherosclerosis, Circ Res 86 (2000), 391-395.

[17] D. Ito, M. Murata, K. Watanabe et al., C242T polymorphism of NADPH oxidase p22PHOX gene and ischemic cerebrovascular disease in the Japanese population, Stroke 31 (2000), 936-939.

[18] O. Stanger, W. Renner, G. Khoschsorur, B. Riglar and T.C. Wascher, NADH/NADPH oxidase p22phox C242T polymorphism and lipid peroxidation in coronary artery disease, Clin Physiol 21 (2001), 718-722.

[19] H. Cai, N. Duarte, D.E. Wilcken and X.L. Wang, NADH/NADPH oxidase p22phox C242T polymorphism and coronary artery disease in the Australian population, Eur $J$ Clin Invest 29 (1999), 744-748.

[20] R. Hayaishi-Okano, Y. Yamasaki, Y. Kajimoto et al., Association of NADPH oxidase p22phox gene variation with advanced cartoid atherosclerosis in Japanese type 2 diabetes, Diabetes Care 26 (2003), 458-463.

[21] S. Matsunaga-Irie, T. Maruyama, Y. Yamamoto et al., Relation between development of nephropathy and the p22phox C242T and receptor for advanced glycation end product G1704T gene polymorphisms in type 2 diabetic patients, Diabetes Care 27 (2004), 303-307.

[22] A.D. Hodgkinson, B.A. Millward and A.G. Demaine, Association of the $\mathrm{p} 22$ phox component of $\mathrm{NAD}(\mathrm{P}) \mathrm{H}$ oxidase with susceptibility to diabetic nephropathy in patients with type 1 diabetes, Diabetes Care 26 (2003), 3111-3115.

[23] G. Wolf, U. Panzer, S. Harendza, U. Wenzel and R.A. Stahl, No association between a genetic variant of the p22(phox) component of $\mathrm{NAD}(\mathrm{P}) \mathrm{H}$ oxidase and the incidence and progression of IgA nephropathy, Nephrol Dial Transplant 17 (2002), 1509-1512.

[24] K. Doi, E. Noiri, A. Nakao et al., Haplotype analysis of $\mathrm{NAD}(\mathrm{P}) \mathrm{H}$ oxidase p22 phox polymorphisms in end-stage renal disease, J Hum Genet 50 (2005), 641-647. 


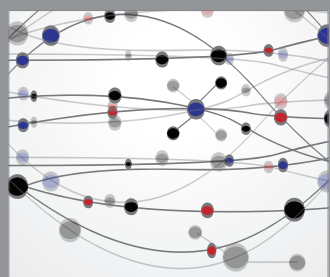

The Scientific World Journal
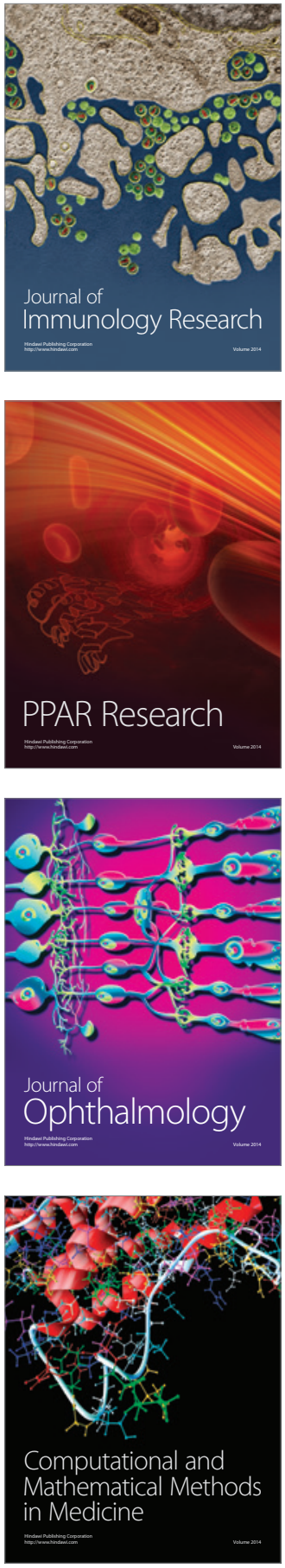

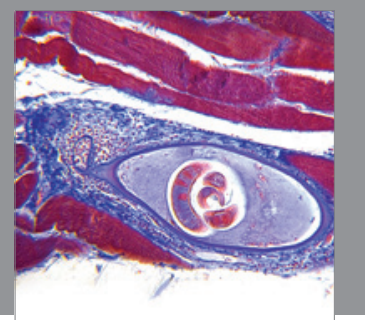

Gastroenterology

Research and Practice
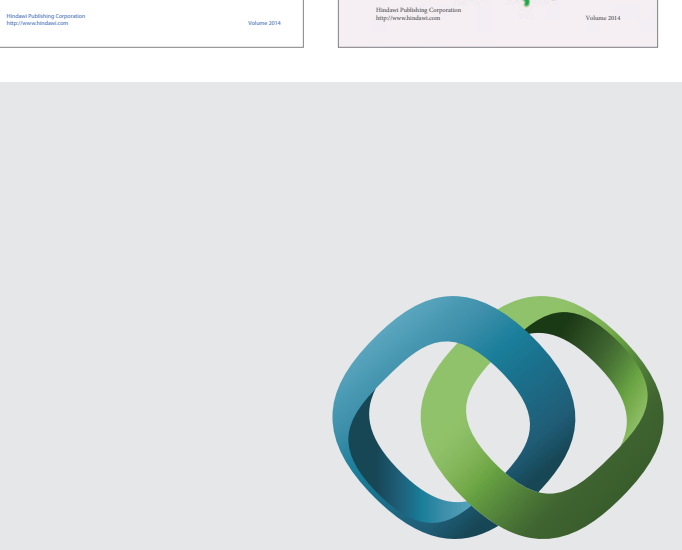

\section{Hindawi}

Submit your manuscripts at

http://www.hindawi.com
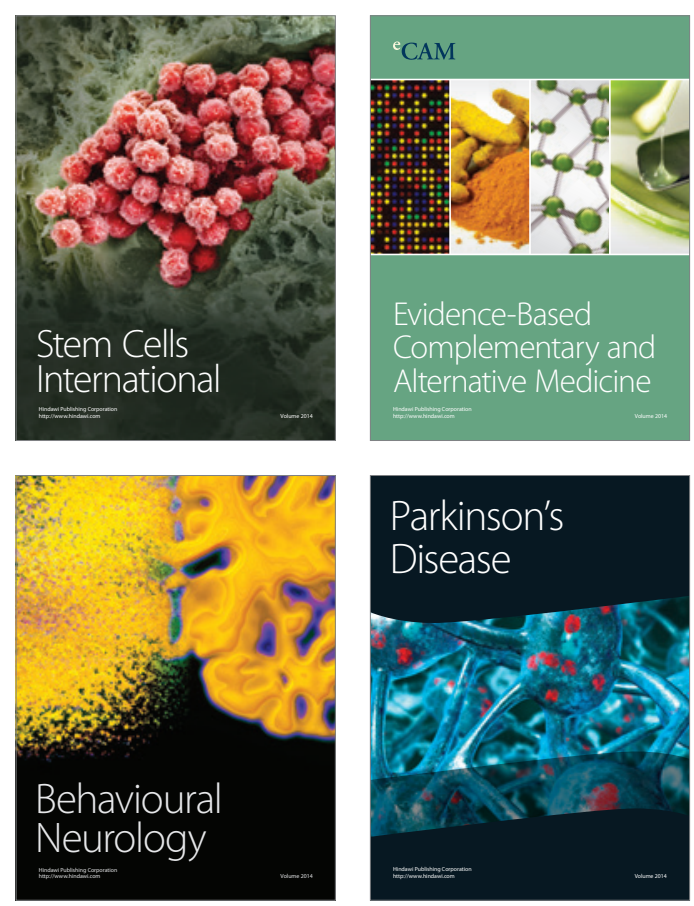

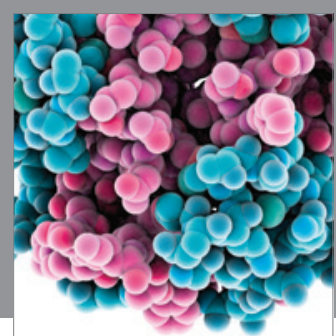

Journal of
Diabetes Research

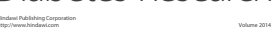

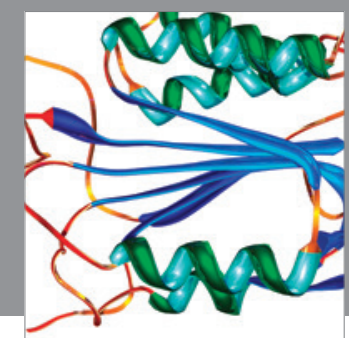

Disease Markers
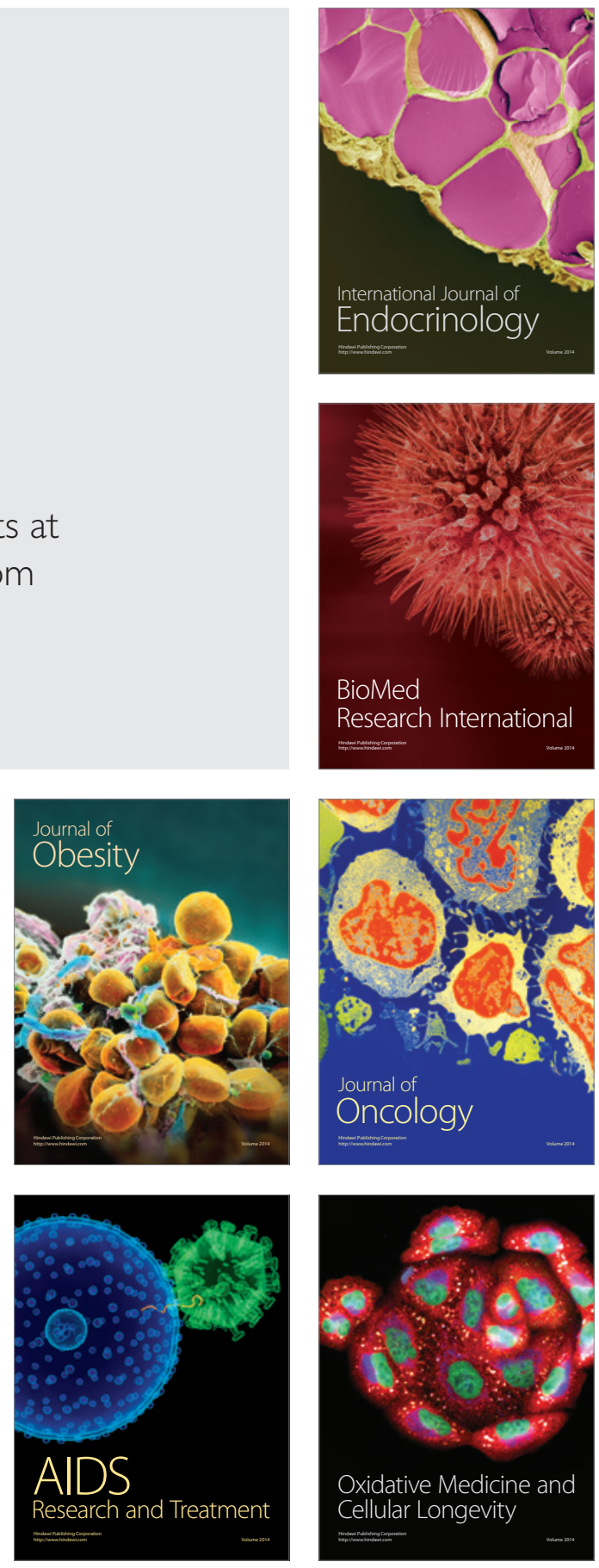